\title{
Teleodontologia em consonância com as Diretrizes Curriculares Nacionais no desenvolvimento de competências profissionais
}

Pablo Guilherme Caldarelli*; Ana Estela Haddad**

* Docente do Departamento de Medicina Oral e Odontologia Infantil, Universidade Estadual de Londrina

** Docente do Departamento de Ortodontia e Odontopediatria, Faculdade de Odontologia, Universidade de São Paulo

\section{RESUMO}

As Diretrizes Curriculares Nacionais (DCN) em Odontologia definem os objetivos do curso assim como os princípios, fundamentos, condições e procedimentos da formação de cirurgiões-dentistas. A Teleodontologia em consonância com as DCN pode ampliar a inserção das instituições de ensino nas ações de capacitação e educação permanente dos profissionais da rede de serviços de saúde, fortalecendo a integração ensino-serviço e ampliando as oportunidades de aplicar metodologias ativas no processo ensinoaprendizagem. O presente estudo tem como objetivo discutir a importância e o impacto da Teleodontologia na formação profissional em Odontologia. Trata-se de um estudo descritivo e exploratório realizado por meio de um levantamento bibliográfico de publicações indexadas nas bases eletrônicas de dados BBO, ISI, LILACS, MEDLINE e SciELO e em documentos oficiais. Concluiu-se que a Teleodontologia pode ser considerada como uma importante ferramenta aplicada à teleassistência e à teleducação, com grande potencial para apoiar a implementação e consolidação das DCN nos cursos de graduação em Odontologia.
Descritores: Educação em Odontologia. Ensino. Currículo. Telemedicina.

\section{INTRODUÇÃO}

A partir da década de 1990, com a regulamentação da Constituição Federal de 1988 e a publicação da Lei $8080 / 90^{1}$, as discussões sobre a formação profissional em saúde no país se intensificaram amplamente ${ }^{2,3}$. Neste cenário, entre os anos de 1998 e 1999, diversas reuniões aconteceram com a finalidade de subsidiar a elaboração das Diretrizes Curriculares Nacionais (DCN) do curso de Odontologia no Brasil, a qual foi publicada a partir do ano de 2002 por meio do Parecer CES/CNE 1.300/2001 ${ }^{4}$, de 06 de novembro de 2001 e a Resolução CNE/CES 3, de 19 de fevereiro $2002^{5}$, publicada no Diário Oficial da União em 4 de março de 2002. A partir desta publicação iniciaram-se tomadas de providências para que as Instituições de Ensino Superior (IES) dessem seus primeiros passos para implementação das DCN nos cursos de Odontologia ${ }^{3}$. 
As DCN em Odontologia definem os objetivos do curso assim como os princípios, fundamentos, condições e procedimentos da formação de cirurgiões-dentistas. Essas diretrizes devem ter aplicação nacional e serem utilizadas como eixo orientador na elaboração dos currículos implementados por todas as instituições de ensino superior, sendo complementadas por uma dimensão diversificada e específica, que reflita a experiência, a vocação de cada escola e as necessidades e imposições da região na qual se situa ${ }^{6,7}$.

A formação em Odontologia sempre esteve pautada prioritariamente no exercício privado da profissão, com ênfase no biologicismo, na sofisticação de técnicas e instrumentos. No entanto, políticas públicas em saúde, como a inserção da saúde bucal na Estratégia de Saúde da Família e o anúncio da prioridade dada pelo governo federal ao programa Brasil Sorridente, fizeram com que os serviços públicos passassem a compor um significativo mercado de trabalho para os profissionais da Odontologia ${ }^{3,6,7}$. Para se ter ideia, a publicação "Perfil Atual e Tendências do Cirurgião-Dentista Brasileiro"8 mostrou que, em 2010, cerca de 1/3 dos mais de 200.000 cirurgiões-dentistas brasileiros possuíam vínculo com o serviço público.

Este cenário, entretanto, defronta-se com a precária disponibilidade de profissionais de saúde dotados de visão humanística e preparados para prestar cuidados contínuos e resolutivos à comunidade, com a atenção primária à saúde funcionando como a porta de entrada do sistema de saúde. Para superação desse obstáculo, ocorreram mudanças no ensino tradicional nas instituições acadêmicas, associadas ao cumprimento das DCN, uma vez que as políticas do Ministério da Saúde, relatam a necessidade de diminuição do distanciamento entre a formação dos profissionais de saúde e as necessidades do Sistema Único de Saúde (SUS) ${ }^{3,6}$. Com esse intuito, desde a criação da Secretaria de Gestão do Trabalho e da Educação na Saúde (SGTES) no Ministério da Saúde e por meio de investimentos consideráveis de recursos, uma diversidade de experiências bemsucedidas tem sido implementada em cursos da área da saúde em todo país ${ }^{9}$.

A era da tecnologia, entre tantas mudanças que tem provocado e em um ritmo nunca antes experimentado, tem permitido o surgimento de novas alternativas com relação aos recursos educacionais, que trazem entre suas características a flexibilização na entrega de conteúdos educacionais, a interatividade envolvendo os atores e inclusive o sistema e o impacto visual. Dessa forma, a Teleodontologia, como campo de conhecimento integrante da Telessaúde, vem evoluindo nos anos recentes, em especial com ênfase na teleducação interativa, na teleassistência e na produção de pesquisas multicêntricas. Ela pode ser definida como o uso das Tecnologias de Informação e Comunicação (TIC) para a troca de dados e informações em saúde, além de prover serviços de saúde em situações em que seja necessário transpor barreiras geográficas, temporais, sociais e culturais ${ }^{10,11}$.

Neste contexto, a Teleodontologia em consonância com as DCN pode ampliar a inserção das Instituições de Ensino Superior (IES) nas ações de capacitação e educação permanente dos profissionais da rede de serviços do SUS, fortalecendo a integração ensino-serviço, prevista nas DCN, além de ampliar as oportunidades de aplicar metodologias ativas no processo ensinoaprendizagem $^{10}$.

Dessa forma, o presente estudo tem como objetivo discutir a importância e o 
impacto da utilização da Teleodontologia e sua participação na formação profissional em Odontologia, refletindo sobre os aspectos potencializadores e dificultadores, tendo em vista as habilidades e competências necessárias à formação profissional na área da saúde e o cumprimento das regulamentações propostas pelas DCN.

\section{METODOLOGIA}

Trata-se de um estudo descritivo e exploratório, no qual a estratégia de identificação e seleção dos estudos foi um levantamento bibliográfico de publicações indexadas nas bases eletrônica de dados BBO, ISI, LILACS, MEDLINE e SciELO, utilizando-se os seguintes descritores em ciências da saúde (DeCS): "Educação em Odontologia", "Ensino", "Currículo" e "Telemedicina".

Foram adotados os seguintes critérios para seleção dos artigos: estudos (descritivos, observacionais, intervencionais, tecnológicos ou revisões da literatura) publicados integralmente em periódicos que adotam a revisão por pares, indexados junto às bases de dados elencadas para a realização do trabalho, em idioma português, inglês ou espanhol. O início do período de abrangência da busca encontra-se baseado na implantação das Diretrizes Curriculares Nacionais (DCN) do curso de Odontologia no Brasil, a qual ocorreu a partir do ano de 2002 (Parecer CES/CNE 1.300/2001, de 06 de novembro de 2001 e Resolução CNE/CES 3, de 19 de fevereiro 2002). Dessa forma, o período compreendeu desde o ano de 2002 até o ano de 2014. Durante a seleção dos estudos, a avaliação dos títulos e dos resumos (abstracts) identificados na busca inicial foi realizada obedecendo rigorosamente aos critérios de inclusão definidos para a pesquisa.
Do material obtido, procedeu-se à leitura minuciosa de cada resumo de artigo científico, destacando aqueles que responderam aos objetivos propostos por este estudo, a fim de organizar e tabular os dados. Para a organização e tabulação dos dados, foi elaborado um instrumento de coleta de dados contendo: título do artigo científico, título do periódico, ano de publicação, país do estudo, categoria do estudo, referencial teórico, método de análise, objetivo e resultados encontrados. Seguindo os critérios de inclusão, foram selecionados para análise os estudos que se encontram referenciados no presente trabalho.

Além disso, foram também consultados documentos oficiais, bem como as legislações do Ministério da Educação e do Ministério da Saúde, relacionadas com a temática em questão.

\section{RESULTADOS E DISCUSSÃO}

A Telessaúde pode ser definida como o conjunto de técnicas, práticas, atitudes, modos de pensar e novos valores que se desenvolvem em consequência do crescimento do espaço digital ${ }^{11}$. Além disso, ela encontra-se diretamente relacionada com a utilização de tecnologias de comunicação para o intercâmbio de informações válidas para diagnóstico, prevenção e tratamento de doenças e a contínua educação de prestadores de serviços em saúde, assim como para fins de pesquisas e avaliações ${ }^{13,14}$.

Trazendo esse conceito e as ferramentas da Telessaúde para a Odontologia, a Teleodontologia tem sido considerada como um método prático e economicamente viável de prover atenção em saúde para grupos populacionais desassistidos, incluindo os indivíduos socialmente desfavorecidos, que moram em 
localizações remotas ou áreas rurais e que não tenham acesso a cuidados odontológicos de rotina ${ }^{15,16}$. A Teleodontologia aborda também os aspectos de teleducação interativa, desenvolvimento de ferramentas e programas de promoção de saúde bucal, supervisão de tratamentos a distância além de teleconsultas com especialistas $^{17,18}$.

A difusão da Teleodontologia no Brasil torna-se uma importante ferramenta para a melhoria da qualidade educacional, uma vez que a Odontologia tem um papel significativo na saúde da população brasileira. Logo, com o auxílio da Teleodontologia, grupos institucionais e universitários podem promover programas nacionais de estímulo à saúde bucal, com a possibilidade de parcerias com órgãos públicos, privados e organizações não-governamentais $(\mathrm{ONG})^{10}$.

Dessa forma, a utilização da Teleodontologia no processo ensinoaprendizagem encontra-se em consonância com os preceitos da Resolução CNE/CES n ${ }^{\circ}$ 3 , de 19 de fevereiro de $2002^{5}$ que instituiu as Diretrizes Curriculares Nacionais para o curso de graduação em Odontologia, objetivando a formação geral e específica dos egressos/profissionais com ênfase na promoção, prevenção, recuperação e reabilitação da saúde ${ }^{4}$. O cirurgião-dentista graduado de acordo com essa resolução deve apresentar uma formação generalista, humanista, crítica e reflexiva para atuar em todos os níveis de atenção à saúde, com base no rigor técnico e científico, sendo capacitado ao exercício de atividades referentes à saúde bucal da população, pautado em princípios éticos, legais e na compreensão da realidade social, cultural e econômica do seu meio, dirigindo sua atuação para a transformação da realidade em benefício da sociedade ${ }^{6}$.

De acordo com Jampani et al. (2011) ${ }^{14}$, a Teleodontologia é uma combinação de telecomunicações e Odontologia envolvendo a troca de informações clínicas e de imagens a distâncias para consulta odontológica e planos de tratamento. Os autores afirmam que a utilização da Teleodontologia tem a capacidade de melhorar o acesso aos cuidados de saúde bucal, melhorando a prestação de cuidados à saúde e diminuindo seus custos. Ainda afirmam que a Teleodontologia tem o potencial de eliminar as disparidades de saúde bucal entre as comunidades rurais e urbanas, mostrando-se como uma importante ferramenta para formação de profissionais generalistas, humanistas, capazes de atuar em todos os níveis de atenção à saúde, como preconizado pelas $\mathrm{DCN}^{5}$.

Chen et al. (2003) ${ }^{19}$ afirmam que, mesmo a Teleodontologia sendo considerada como um campo relativamente novo, ela pode mudar substancialmente a dinâmica dos sistemas de atendimento e a formação odontológica. Os autores relatam que Teleodontologia oferece novas oportunidades para a educação em Odontologia, fornecendo um fácil acesso aos profissionais da atenção primária, ajudando assim na educação e na realização de programas de educação continuada em Odontologia, aspecto de importância também regulamentado pelas DCN como competência geral a ser desenvolvida por um cirurgião-dentista ${ }^{5}$.

A Lei Orgânica $8080 / 90^{1}$, em seu artigo $n^{\circ} 27$ reconhece que os serviços públicos que integram o SUS constituem um campo de práticas para o ensino e a pesquisa, de modo a articular os interesses das Instituições de Educação Superior (IES) e do SUS, com vistas à melhoria da qualidade do atendimento à população ${ }^{2}$. As DCN dos cursos de graduação na área da saúde, definidas para todos os cursos desta área, apontam que "a formação do profissional deve contemplar o sistema de saúde vigente 
no país, o Sistema Único de Saúde (SUS), baseado na atenção integral da saúde em um sistema regionalizado e hierarquizado de referência e contrarreferência e no trabalho em equipes" $"$.

Neste importante contexto, Haddad e Skelton-Macedo $\quad(2012)^{10}$ relatam a importância da Teleodontologia na ampliação da inserção da IES nas ações de capacitação e educação permanente dos profissionais da rede de serviços do SUS, fortalecendo a integração ensino-serviço, também prevista pelas DCN dos cursos de graduação em Odontologia. Além disso, as autoras reafirmam o papel dessa tecnologia no ensino, como uma forma de ampliação das oportunidades de aplicar metodologias ativas no processo ensino-aprendizagem.

Macedo et al. $(2012)^{20}$ relatam a Teleodontologia como uma ciência inovadora que oferece conhecimento odontológico em dois âmbitos principais: a teleassistência e a teleducação. Os autores afirmam que por meio da utilização dessas inovações tecnológicas, torna-se possível a aplicação desse conhecimento em diversos âmbitos da vida profissional, como uma nova visão de clínica interdisciplinar. Além disso, os cuidados devidos à manutenção do sigilo das informações relativas aos pacientes e formas seguras e criativas de se incorporar as Tecnologia de Informação e Comunicação (TIC) no dia a dia do cirurgião-dentista tornam-se cada vez mais concretas, acrescentando valor às ações profissionais, qualificação na atenção à saúde bucal brasileira e melhorias na educação/formação em Odontologia.

Neste sentido, podemos exemplificar a utilização dessas tecnologias por meio das práticas de teleconsultoria e segunda opinião formativa. A primeira surge como uma espécie de diálogo, por meio de instrumentos de telecomunicação, entre profissionais da área da saúde onde são esclarecidas dúvidas sobre procedimentos clínicos, podendo ser síncrona ou assíncrona. Já a segunda opinião formativa pode ser definida como a unidade composta por perguntas e respostas, resultantes de uma teleconsultoria, que passou por avaliação por pares, quanto à sua relevância na atenção primária à saúde, e na qual a resposta produzida baseou-se em levantamento bibliográfico, destacando as melhores evidências científicas e clínicas disponíveis sobre o assunto ${ }^{21}$.

Para Cartes-Velasquez e Bustos-Leal $(2012)^{11}$, os avanços tecnológicos e as TIC têm influenciado as práticas de saúde de maneira substancial, o que tem facilitado o surgimento de um novo modo de "cuidado". Dessa forma, os autores apontam a Teleodontologia como uma importante e potente ferramenta no auxílio da superação de desafios relacionados com a prática-clínica, o ensino e a formação em Odontologia e também como apoio à saúde bucal na atenção primária dos serviços de saúde.

No entanto, embora o uso das TIC já esteja bastante disseminado com resultados bem documentados na área da saúde, tanto na dimensão educacional como de pesquisa e assistencial, observa-se ainda manifestações de resistência e reserva quanto à sua utilização por parte dos docentes. Entre as preocupações apontadas, encontra-se o desafio da formação na área da saúde, que tem suas bases estabelecidas no aprendizado não apenas teórico, mas de habilidades, competências e atitudes, que exigem treinamento prático, aliado a supervisão por docentes ou por profissionais mais experientes. Essa resistência justifica-se no argumento de que não é possível realizar estas atividades por meio da educação à distância. Além disso, surge também como resistência o uso 
indiscriminado das TIC, de forma acrítica e pouco apropriada, ao invés de tê-la como coadjuvante de um projeto pedagógico bem fundamentado ${ }^{21}$.

Assim, torna-se relevante compreender que não se trata de optar pela educação a distância em detrimento da educação presencial, já que a denominação "educação a distância" talvez não seja apropriada para designar o uso que tem sido feito das TIC. Estas devem ser compreendidas como ferramentas de apoio e complementação, a serem utilizadas num contexto planejado e estruturado de abordagem metodológica suportada por uma linha pedagógica própria ${ }^{21}$.

Dessa forma, as TIC podem ser consideradas como uma potente ferramenta, seja na teleassistência ou na teleducação. No entanto, deve-se levar em consideração que estas não possuem caráter substitutivo às práticas-clínicas, às necessidades de fortalecimento das redes de atenção e principalmente, no caso da teleducação, ao planejamento político pedagógico.

\section{CONCLUSÕES}

A Teleodontologia torna-se uma importante ferramenta aplicada à teleassistência e à teleducação, com grande potencial para apoiar a implementação e consolidação das Diretrizes Curriculares Nacionais (DCN) nos cursos de graduação em Odontologia, podendo ser considerada como uma excelente proposta de integração entre tecnologias e educação, incentivando e auxiliando a articulação entre o ensino, a pesquisa e a extensão.

Além disso, a Teleodontologia pode ampliar a inserção das Instituições de Ensino Superior (IES) nas ações de capacitação e educação permanente dos profissionais da rede de serviços do SUS, fortalecendo a integração ensino-serviço e ampliando as oportunidades de se aplicar metodologias ativas no processo de ensino-aprendizagem.

\section{ABSTRACT \\ Teledentistry in consonance with the National Curriculum Guidelines in the development of professional competencies} The National Curriculum Guidelines (DCN) for Dentistry define the course objectives, the principles, fundamentals, conditions and procedures of the dentistry formation. The Teledentistry in consonance with the DCN can enlarge the insertion of educational institutions in training activities and permanent education of health services network professionals, strengthening the teaching-service integration and broadening opportunities to apply active methods in the teaching process learning. This study aims to discuss the importance and the impact of Teledentistry in dentistry professional training. This is a descriptive and exploratory study realized through a literature review of publications indexed in electronic databases BBO, ISI, LILACS, MEDLINE and SciELO and official documents. It was concluded that the Teledentistry is an important tool applied to teleassistance and teleducation, with great potential to support the implementation and consolidation of DCN in Dentistry undergraduate courses.

Descriptors: Education, Dental. Teaching. Curriculum. Telemedicine.

\section{REFERÊNCIAS}

1. Brasil. Lei $\mathrm{n}^{\circ} 8080$, de 19 de setembro de 1990. Dispõe sobre as condições para a promoção, proteção e recuperação da saúde, a organização e o funcionamento dos serviços correspondentes e dá outras providências. Diário Oficial da União. Brasília, 20 set. 1990; Seção 1.

2. Cavalheiro MTP, Guimarães AL. Formação para o SUS e os desafios da 
integração ensino serviço. Caderno FNEPAS. 2011; 1(1):1-9.

3. Morita MC, Kriger L, Carvalho ACP, Haddad AE. Implantação das Diretrizes Curriculares Nacionais em Odontologia [Internet]. Maringá: Editora Dental Press. 2007 [Acesso em: 07/03/2016]. Disponível em: http://abeno.org.br/arqui vos/downloads/download_201111091533 52.pdf

4. Brasil. CNE. Parecer CES/CNE 1300/2001 [internet]. Diário Oficial da União, Seção 1, p. 25; Brasília, 7 de dezembro de 2001- [Acesso em: 07/03/2016]. Disponível em: http:// portal.mec.gov.br/cne/arquivos/pdf/CES1 300.pdf

5. Brasil. CNE. Resolução CNE/CES 3/2002 [internet]. Diário Oficial da União, Seção 1, p.10; Brasília, 4 de março de 2002 [Acesso em: 07/03/2016]. Disponível em: http://portal.mec.gov.br/cne/arquivos/pdf/ CES032002.pdf

6. Carvalho ACP. Planejamento do curso de graduação de Odontologia: É importante planejar os cursos de graduação considerando-se as Diretrizes Curriculares Nacionais. Rev ABENO. 2004; 4(1):7-13.

7. Fonseca EP. As Diretrizes Curriculares Nacionais e a formação do cirurgiãodentista brasileiro. J Manag Prim Health Care. 2012; 3(1):158-178.

8. Morita MC, Haddad AE, Araújo ME. Perfil atual e tendências do cirurgiãodentista brasileiro. Maringá: Dental Press. 2010 [Acesso em: 07/03/2016]. Disponível em: http://cfo.org.br/wpcontent/uploads/2010/04/PERFIL_CD_B R_web.pdf

9. Haddad AE, Brenelli S, Passarella TM, Campos T. Política Nacional de Educação na Saúde. Rev Baiana Saúde Pública. 2008; 32(supl 1):98-114.
10. Haddad AE, Skelton-Macedo MC. Teleodontologia na formação dos profissionais de saúde. In: Mathias I, Monteiro A. (Org.). Gold book: Inovação tecnológica em educação e saúde. Rio de Janeiro: EdUERJ; 2012 [Acesso em: 07/03/2016]. Disponível em: http:// www.telessaude.uerj.br/resource/goldboo k/pdf/12.pdf

11. Cartes-Velasquez R, Bustos-Leal A. Teleodontología: Conceptos, experiencias y proyecciones. Odontoestomatología. 2012; 14(20):17-25.

12. Haddad AE, Bonecker M, SkeltonMacedo MC. Research in the field of health, dentistry, telehealth and teledentistry. Braz Oral Res. 2014; 28(1) $: 1-2$.

13. Wen CL. Telemedicina e Telessaúde - Um panorama no Brasil. Informática Pública. 2008; 10(8):07-15.

14. Correia ADMS, Dobashi BF, Gonçalves CCM, Monreal VRFD, Nunes EA, Haddad PO, et al. Teleodontologia no Programa Nacional Telessaúde Brasil Redes: Relato da experiência em Mato Grosso do Sul. Rev ABENO. 2014; 14(1):17-29.

15. Jampani ND, Nutalapati R, Dontula BSK, Boyapati R. Applications of teledentistry: A literature review and update. J Int Soc Prevent Communit Dent. 2011; 1(1):3744.

16. Daniel SJ, Wu L, Kumar S. Teledentistry: A systematic review of clinical outcomes, utilization and costs. J Dent Hyg. 2013; 87(6):345-352.

17. Torres-Pereira C, Possebon RS, Simões A, Bortoluzzi MC, Leão JC, Giovanini AF, Piazetta CM. E-mail for distance diagnosis of oral diseases: a preliminary study of teledentistry. J Telemed Telecare 2008; 14(8):435-8. 
18. Olival ARB, Curvino M, Faria M, Groisman S. Teleodontologia: Um novo horizonte. Perionews. 2008; 2(4):311315.

19. Chen JW, Hobdell MH, Dunn K, Johnson KA, Zhang J. Teledentistry and its use in dental education. J Am Dent Assoc. 2003; 134(3):342-346.

20. Macedo MCS, Jacob CH, Ramos DLP, Cardoso RJA; Antoniazzi JH. Teleodontologia: Valores agregados para o clínico/especialista. Rev Assoc Paul Cir Dent. 2012; 66(2):95-99.
21. Haddad AE. A Odontologia na Política de Formação dos Profissionais de Saúde, o papel da Teleodontologia como ferramenta do processo de ensinoaprendizagem e a criação do Núcleo de Teleodontologia da FOUSP [Tese de Livre Docência]. São Paulo: Faculdade de Odontologia da Universidade de São Paulo - FOUSP.

Correspondência para:

Pablo Guilherme Caldarelli

e-mail: pablocaldarelli@hotmail.com

Rua Juiz de Fora, ${ }^{\circ} 230$

86062-680 Jardim Champagnat, Londrina, PR 